\title{
Preparation and properties of silicone fouling release coatings with long- life afterglow fluorescent
}

\author{
Zhanping Zhanga, Hao Li and Yuhong Qi \\ Department of Materials Science and Engineering, Dalian Maritime University, Dalian 116026, China
}

\begin{abstract}
Based on polydimethylsiloxane, three-component coatings were prepared with different content of luminescence powder. The results showed that the illuminance of coatings increases with the content of luminescence powder, decays exponentially with the afterglow time, increases exponentially with the increase of exposure time. The afterglow illuminance augments with irradiated light illuminance. All coatings are hydrophobic and oleophilic. Surface free energy decreases with the increase of luminescence powder. They have highest impact-resistance and bend flexibility. The luminescence powder does not change obviously the shore hardness, tensile breaking strength, breaking elongation rate, elastic modular and roughness of coatings. The static test panels in sea generally could be covered obviously by biofouling including sponges, bryophytes and mussels, hydra, kelp, green algae after 2 months of immersion during growing season. But it never found that the barnacle attached on the coating surface during 4 years of immersion test. The static anti-fouling ability of the coatings is very limited. In addition, the sea creatures attached on the coating surface can be easily removed; even attached organisms will fall off and expose again the smooth coating surface. Consequently, all coatings with long-life afterglow fluorescent have a significant effect on preventing adhesion of barnacle and fouling-release performance.
\end{abstract}

\section{Introduction}

All man-made structures in marine environments suffer from the problem of biofouling that the unwanted growth of bacteria, algae and invertebrates. It causes extra financial burdens, excessive consumption of energy, and contamination of the environment. Current self-polishing antifouling coating technologies derived from copperbased compounds, could induce detrimental effects on marine environment [1]. Hence there is an exigent need for developing efficient and non-toxic marine coatings that would possess no ecological concern.

During past years, more researches were focused on the non-toxic antifouling coatings, especially on fouling release coatings. Fouling release coatings present a surface which can reduce the adhesion strength of fouling organisms enabling cleaning by pressurized water jets or in some cases by hydrodynamic forces generated as the ship sailing [2]. These include silicone and fluoropolymer coatings. Amount of studies has been conducted on fouling-release coating systems based on polydimethylsiloxane (PDMS), appear to out-perform fluoropolymers and be a viable fouling-release coating system. Surface properties of the PDMS coatings including surface free energy, elastic modulus and thickness are considered be important to the fouling release performance [3]. In addition, some researchers pointed out that incorporation of low molecular weight silicone oils into silicone coatings improves their fouling release properties [4].

Biofouling process occurs on the interface between seawater and coating surface. Previous researches showed that the colour and brightness of coating surface can affect the attachment behaviour of marine biofouling [1]. Furthermore, invertebrates like barnacle, adhere to all kinds of surfaces in the worldwide ocean and the animals settle as larval cyprids, prefer live in dark environment [2]. Considering there is sunlight undersea in the depth of $200 \mathrm{~m}$, we therefore conceived to develop a novel technique that long afterglow luminous coating will be used to antifouling in marine environment in previous study. The results have proved that phosphorescent materials in coating can be activated by sunlight during day even the coating was immersed in seawater [5]. In addition, Cao's investigation has proven that the luminescence of coating during night will disturb the normal physiological activity and metabolism of marine organism, and provoke larval cyprids to keep away from the coating [6].

In this paper, based on PDMS, five fouling release coatings were prepared consisting of 107 silicone gum, dimethyl silicone oil, tetraethylorthosilicate as crosslinked curing agent, heavy calcium carbonate as filler, alkali soils silicon aluminate as luminescence powder, dibutyltin dilaurate as catalyst. The aim of this study is to evaluate the influence of luminescence powder

a Corresponding author: zzp@dlmu.edu.cn 
content on the properties of silicone fouling release coatings with long-life afterglow fluorescent.

\section{Materials and methods}

\subsection{Preparation of coatings}

Based on polydimethylsiloxane (PDMS), five threecomponent paints were prepared with controlling the content of luminescence powder (Alkali soils silicon aluminate, body colour is light-yellow; glowing colour is blue) respectively $2.5,5,7.5,10,12.5$ wt. \%. Component A included PDMS, silicone oil, fillers, luminescence powder and additives. TEOS mixed with xylene to make curing agent (Component B), the mixture of DBTDL and acetylacetonate was prepared into catalytic agent (Component C). The dimethyl silicone oil (DY-201-100), which formula is $\left(\mathrm{CH}_{3}\right)_{3} \mathrm{SiO}\left[\left(\mathrm{CH}_{3}\right)_{2} \mathrm{SiO}\right] \mathrm{n}-\mathrm{Si}\left(\mathrm{CH}_{3}\right)_{3}$. The viscosity is $100 \pm 5\left(25^{\circ} \mathrm{C}\right) \mathrm{mPa} \cdot \mathrm{s}$, was bought from Shandong Dayi Chemical Co. Ltd. The 107 silicone gum (DY-OH501), which formula is $\mathrm{HO}-\mathrm{Si}\left(\mathrm{CH}_{3}\right)_{2} \mathrm{O}\left(\mathrm{Si}\left(\mathrm{CH}_{3}\right)_{2}\right.$ $\mathrm{O})_{n} \mathrm{Si}\left(\mathrm{CH}_{3}\right)_{2}-\mathrm{OH}$, possesses the reactivity of hydroxyl group that can be made into some crosslinked as elastomer coating. The tetraethylorthosilicate (TEOS) is crosslinked curing agent, the dibutyltin dilaurate (DBTDL) is catalyst. Both of them and xylene were bought from Tianjin Kemiou Chemical Reagent Co., Ltd. BYK-161 and BYK-066N is respectively dispersing agent and defoamer produced by BYK-Chemie GmbH. The composition of studied paints is listed in Table 1. The component A is first mixed at 4000rpm in highspeed stirrer, then grinded to $40 \mu \mathrm{m}$ by cone mill. Three components were mixed for 15 minutes at $\mathrm{A}: \mathrm{B}: \mathrm{C}=18.3: 4: 1$ before painting, then cured in ambient for at least 7 days before tests.

Paint was brushed on glass slides with dimensions of $75 \mathrm{~mm} \times 25 \mathrm{~mm} \times 1 \mathrm{~mm}$, and also cast into a Teflon mould with dimensions of $150 \mathrm{~mm} \times 150 \mathrm{~mm} \times 3 \mathrm{~mm}$. The slide samples were for contact angle and surface characteristics measurement of coatings. Cast specimen was cut strip with $40 \mathrm{~mm} \times 250 \mathrm{~mm}$ for mechanical properties tests. Each coating was prepared three pieces of panels for antifouling immersion test in ocean. The steel plate samples with $300 \mathrm{~mm} \times 200 \mathrm{~mm} \times 3 \mathrm{~mm}$ were in turn coated with epoxy anti-rust primer, intermediate anti-corrosive paint and prepared silicone fouling release coatings with long-life afterglow fluorescent.

\subsection{Mechanical properties}

Tensile samples were tested on a Labthink XLM auto tensile tester. Elastic modulus was fitted with the data which the strain is less than $0.02 \mathrm{~mm} / \mathrm{mm}$. HT220 shore hardness tester was applied to test the hardness of coating.

\subsection{Fluorescence of the coatings}

After some period exposure of the panels under artificial illumination in HPG-280B container, lamp-house was turned off and the fluorescence of the coatings was measured with illuminometer ST-900 at regular intervals.

Table 1. Composition of studied paint.

\begin{tabular}{|c|c|c|c|c|c|c|}
\hline \multirow{2}{*}{\multicolumn{2}{|c|}{\begin{tabular}{|c|} 
Component \\
Composition / wt. $\%$ \\
\end{tabular}}} & \multicolumn{5}{|c|}{ Paint } \\
\hline & & P5 & P10 & P15 & P20 & $\mathrm{P} 25$ \\
\hline \multirow{7}{*}{ A } & $\begin{array}{l}\text { Heavy calcium } \\
\text { carbonate }\end{array}$ & 10 & 7.5 & 5 & 2.5 & 0 \\
\hline & $\begin{array}{c}\text { Luminescence } \\
\text { powder } \\
\text { (PLO-7C) }\end{array}$ & 2.5 & 5 & 7.5 & 10 & 12.5 \\
\hline & $\begin{array}{c}\text { PDMS } \\
(\mathrm{DY}-107)\end{array}$ & \multicolumn{5}{|c|}{50} \\
\hline & Xylene & \multicolumn{5}{|c|}{10} \\
\hline & Fillers & \multicolumn{5}{|c|}{7.5} \\
\hline & Additive & \multicolumn{5}{|c|}{2.5} \\
\hline & $\begin{array}{l}\text { Silicone Oil } \\
\text { (DY-201) }\end{array}$ & \multicolumn{5}{|c|}{2.5} \\
\hline & B & \multicolumn{5}{|c|}{12} \\
\hline & $\mathrm{C}$ & \multicolumn{5}{|c|}{3} \\
\hline & Total & \multicolumn{5}{|c|}{100} \\
\hline
\end{tabular}

\subsection{Surface morphology and topography}

The morphology was investigated by confocal laser scanning microscopy (CLSM) OLS4000 from Olympus. Roughness was measured with software LEXT. The objective lens is used respectively BF plan semiapochromat and LEXT-Dedicated plan apochromat. The coating was observed respectively after curing 7 days, 30 days, 180 days and 360 days.

\subsection{Surface characteristics of the coatings}

Contact angle measurements were conducted using the sessile drop method on a JC2000C (Zhongchen, P. R. China) contact angle measuring system. The surface free energy was calculated based on the contact angle measurements of deionized water and diiodomethane.

\subsection{Antifouling performances of the coatings}

The antifouling property of the samples in the marine environment was also investigated. Panel samples for antifouling test in sea were spread onto $300 \mathrm{~mm} \times 200 \mathrm{~mm} \times 3 \mathrm{~mm}$ steel plate, pre-coated with epoxy anti-rust primer and iron oxide red epoxy intermediate coating. Then panel samples were hung on a floating dock in Lvshun Port in China, and the panels were immersed in the Yellow Sea at depths of 1-2 $\mathrm{m}$ form April 2012. The samples were checked regularly to monitor the progress of the fouling process.

\section{Results and discussion}

\subsection{Illuminance}


The results showed that the illuminance of coatings increases with the content of luminescence powder, the illuminance of every coating decays exponentially with the increase of afterglow time. With the increase of exposure time, the illuminance of coating increases exponentially. When the coating was irradiated under higher light illuminance, the afterglow illuminance of coating augments.

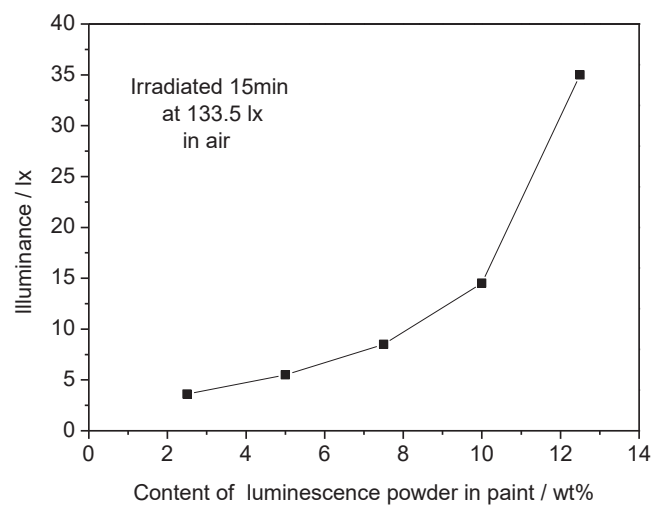

Figure 1. Illuminance of coatings vs. content of luminescence powder.

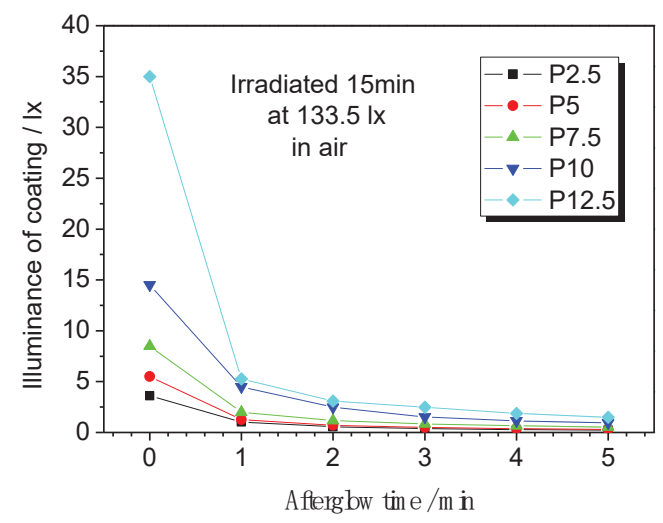

Figure 2. Illuminance of coatings vs. afterglow time.

\subsection{Surface morphology and topography}

Because both of PDMS and silicone oil have very low surface tension, studied paints have excellent levelling property. Cured coating looks very smooth. Morphology of studied coating showed in Figure 3. Measured roughness $\mathrm{Ra}$ of coatings changes from $0.13 \mu \mathrm{m}$ to $0.17 \mu \mathrm{m}$, it is not related to the content of luminescence powder.

\subsection{Surface characteristics}

The surface free energies and components were calculated from contact angle measurements, using the acid-base theory for solids according to the van OssChaudhury-Good approach [6]. The test liquids were distilled water and diiodomethane. The contact angles, the surface free energies and their corresponding polar and dispersive components of the samples were listed in Table 2.The results showed that the water contact angle of coatings is between $103^{\circ}$ and $110^{\circ}$, they are hydrophobic. $\mathrm{CH}_{2} \mathrm{I}_{2}$ contact angle of coatings increases, it showed be oleophilic. Surface free energy of coatings, ranged from $29 \mathrm{~mJ} / \mathrm{m}^{2}$ to $27 \mathrm{~mJ} / \mathrm{m}^{2}$, decrease with the increase of luminescence powder. As shown in Figure 4, it was found that the surface free energy not only results from its dispersive component, but also is related obviously to the dispersive component.

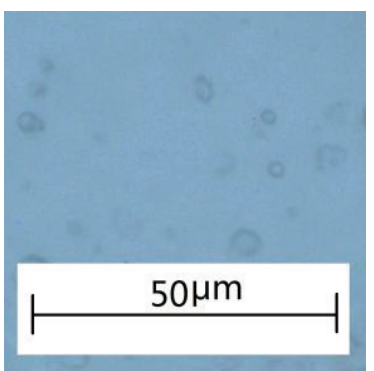

Figure 3. Morphology of studied coating.

Table 2. Contact angle and surface free energy of coatings.

\begin{tabular}{|c|c|c|c|c|c|}
\hline & \multicolumn{2}{|c|}{ Contact angle $/{ }^{\circ}$} & \multicolumn{3}{|c|}{ Surface energy / $\mathrm{mJ} / \mathrm{m}^{2}$} \\
\hline Coating & $\mathrm{H}_{2} \mathrm{O}$ & $\mathrm{CH}_{2} \mathrm{I}_{2}$ & Polar & Dispersive & Total \\
\hline P2.5 & 103.6 & 59.9 & 0.16 & 28.70 & 28.85 \\
\hline P5 & 109.6 & 62.4 & 0.01 & 28.01 & 28.02 \\
\hline P7.5 & 108.2 & 62.9 & 0.01 & 27.48 & 27.49 \\
\hline P10 & 108.0 & 63.0 & 0.01 & 27.39 & 27.40 \\
\hline P12.5 & 110.8 & 64.3 & 0.01 & 26.98 & 27.00 \\
\hline
\end{tabular}

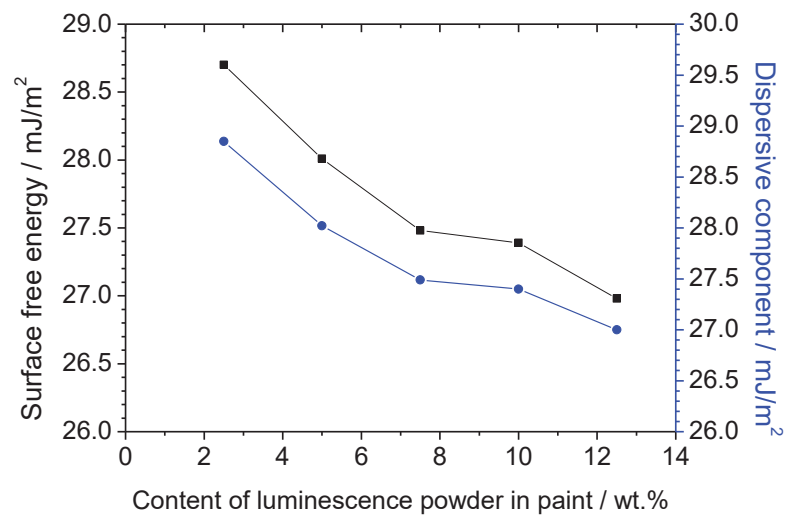

Figure 4. Surface free energy and its dispersive component.

\subsection{Mechanical properties}

All coatings have highest impact-resistance and bend flexibility, they are respectively $50 \mathrm{~kg} \cdot \mathrm{cm}$ and $1 \mathrm{~mm}$. The shore hardness, tensile breaking strength, breaking elongation rate and elastic modular of coatings were listed in Table 3. They are not affected by the content of luminescence powder. The shore hardness is about 27 
HA. The breaking elongation rates of coatings are range from $180 \%$ to $240 \%$. The elastic modular is much low, it is about $0.2 \mathrm{MPa}$. Therefore the coatings showed good elastic deformation capacity.

Table 3. Mechanical properties of coatings.

\begin{tabular}{|c|c|c|c|c|}
\hline Coating & $\begin{array}{c}\text { Breaking } \\
\text { strength } \\
(\mathrm{MPa})\end{array}$ & $\begin{array}{c}\text { Elongation } \\
\text { rate (\%) }\end{array}$ & $\begin{array}{c}\text { Elastic } \\
\text { modular } \\
(\mathrm{MPa})\end{array}$ & $\begin{array}{c}\text { Shore's } \\
\text { hardness } \\
\text { HA (\%) }\end{array}$ \\
\hline P2.5 & 1.17 & 237.88 & 0.23 & 28.8 \\
\hline P5 & 1.08 & 213.33 & 0.20 & 25.8 \\
\hline P7.5 & 1.07 & 235.33 & 0.20 & 27.3 \\
\hline P10 & 0.96 & 179.00 & 0.26 & 28.7 \\
\hline P12.5 & 1.21 & 238.70 & 0.23 & 24.9 \\
\hline
\end{tabular}

\subsection{Antifouling performance}

Because the coatings are non-toxic, as shown in Figure 5, the coating surfaces of static test panels generally could be covered obviously by biofouling after 2 months of immersion in sea during growing season for sea creatures. The marine organisms found on the surface of the coating were sponges, bryophytes and mussels, Hydra, kelp, green algae. But it never found that the barnacle attached on the coating surface during 4 years of immersion test in sea. The static anti-fouling ability of the coatings is very limited. In addition, the sea creatures attached on the coating surface can be easily removed, even attached organisms will fall off and expose again the smooth coating surface. As shown in Figure 6, taking coating P12.5 as an example, the coatings repeatedly undergo organism adhering and fouling, fouling-released and selfcleaning. Consequently, all coatings with long-life afterglow fluorescent have a significant effect on preventing adhesion of barnacle and good fouling-release performance.

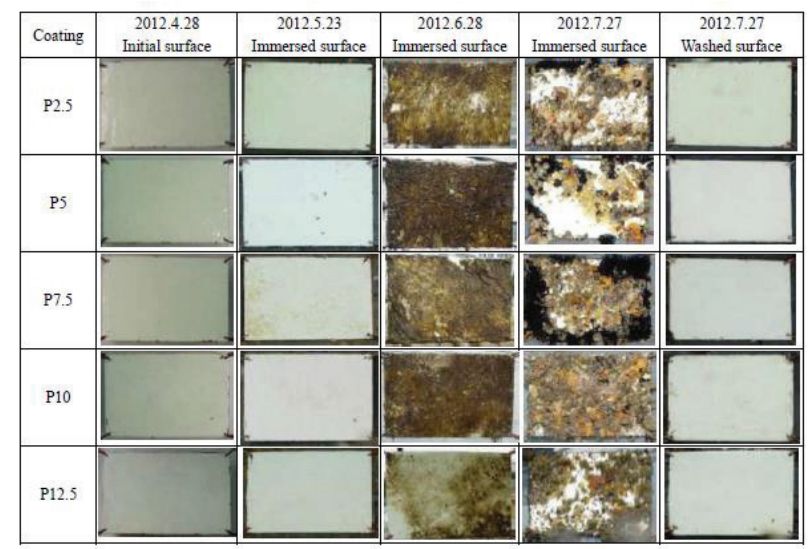

Figure 5. Photos of the coatings for static test panels.

\subsection{Discussion}

Above-mentioned results appear that the antifouling property of coatings is neither related to illuminance nor surface free energy of coatings. This is consistent with the results of previous studies on silicone fouling-release coatings. In other words, the attachment of fouling organisms to such coatings may be related with surface free energy, but not positively correlated [7]. It can also be related to the elastic modulus of the coatings and other factors (e.g., polarity, electronegativity, thickness and smoothness). Specially, it was experimentally confirmed that adhesion is proportional to the square root of the product of elastic modulus (E, MPa) and surface energy $\left(\gamma, \mathrm{mJ} / \mathrm{m}^{2}\right)[8,9]$. Accordingly, the square root of the product can be defined as the relative adhesion factor. This factor used to as a key reference for laboratory design and for selecting fouling-release coatings. Calculated values of relative adhesion factor for studied coatings were listed in Table 4. Clearly, the antifouling performance of studied coatings is not well proportional to the relative adhesion factor. It means that the antifouling performance of the coatings is not only related to single physical parameter, but influenced by some physical and structure factors.

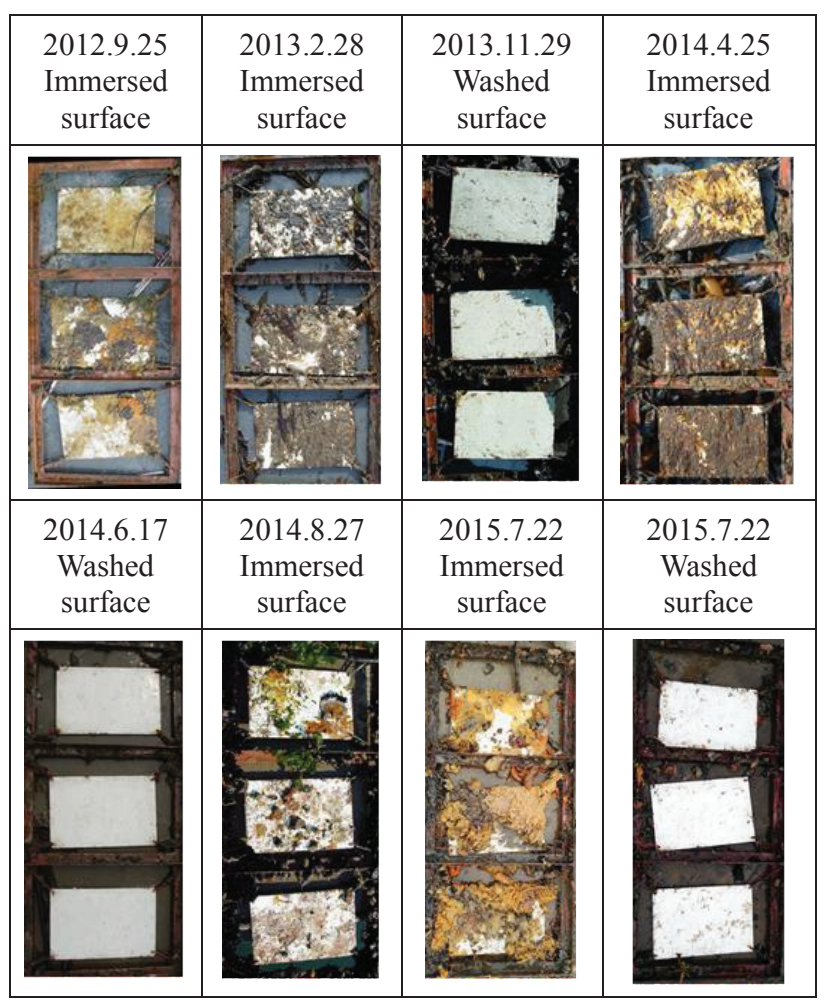

Figure 6. Photos of coating P12.5 for static test panels.

Table 4. Relative adhesion of coatings.

\begin{tabular}{|c|c|c|c|c|c|}
\hline Coating & P2.5 & P5 & P7.5 & P10 & P12.5 \\
\hline $\begin{array}{c}\text { Relative } \\
\text { adhesion factor }\end{array}$ & 2.60 & 2.39 & 2.34 & 2.64 & 2.50 \\
\hline
\end{tabular}

In addition, non-reactive silicone oil was added to the silicone coating in this study. Observation results under confocal laser scanning microscopy showed that no silicone oil appears on the surface of coating samples exposed in air for 180 days. Leached silicone oil was observed on the coating samples exposed in air for 360 
days, as shown in Figure 7. It's clearly that the amount of leached silicone oil increases with the content of luminescence powder. This can be attributed to the effect of oil absorption of luminescence powder and fillers.

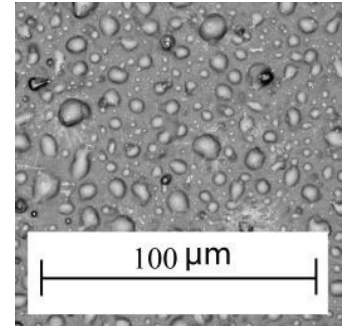

(a) Coating P2.5

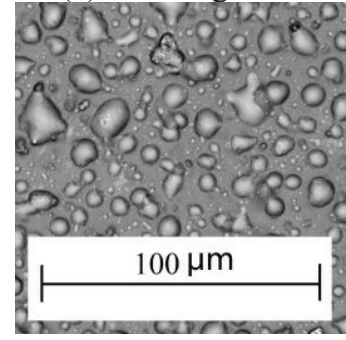

(c) Coating P7.5

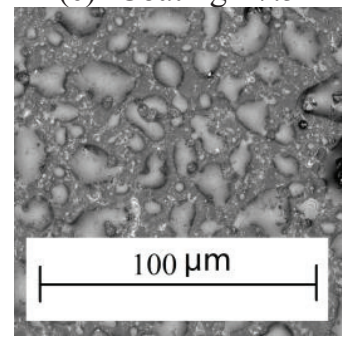

(e) Coating P12.5
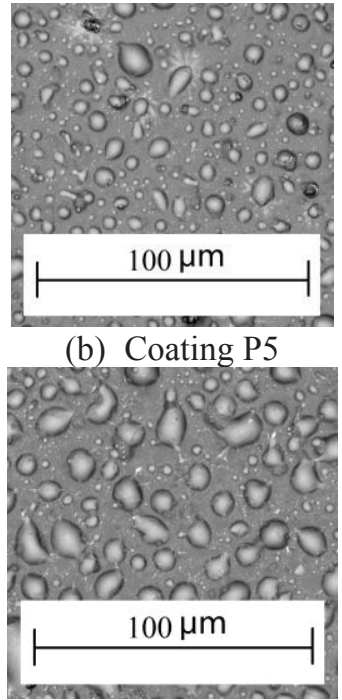

(d) Coating P10 (b) Coating P5

Figure 7. The coating surfaces exposed in air for 360 days.

Above-mentioned results showed that dimethyl silicone oil leach enough slowly from studied coating in air. However leaching rate of silicone oil will accelerate once the coating was immersed in seawater. This has been proven in previous study, relative details can be found in reference [10]. Leached silicone oil will weaken the adhesion between the attached organism and the coating surface. As shown in Figure 5, when the attached organisms accumulate and silicone oil leach to a certain extent, the attached organism will spontaneously fall off. Consequently, studied coatings have good self-cleanly and fouling-release performance.

\section{Conclusions}

The illuminance of coatings increases with the content of luminescence powder, decays exponentially with the afterglow time, increases exponentially with the increase of exposure time. The afterglow illuminance augments with irradiated light illuminance. All coatings are hydrophobic and oleophilic. Surface free energy decreases with the increase of luminescence powder. They have highest impact-resistance and bend flexibility. The luminescence powder does not change obviously the shore hardness, tensile breaking strength, breaking elongation rate, elastic modular and roughness of coatings. The static test panels in sea generally could be covered obviously by biofouling including sponges, bryophytes and mussels, hydra, kelp, green algae after 2 months of immersion during growing season. But it never found the barnacle attached on the coating surface during 4 years of immersion test. The dimethyl silicone oil can leach slowly from studied coating. It will weaken the adhesion between the attached organism and the coating surface. Through the static anti-fouling ability of the coatings is very limited, however, the marine organism attached on the coating surface can be easily removed, even will fall off and expose again the smooth coating surface. Consequently, all coatings with long-life afterglow fluorescent have a significant effect on preventing adhesion of photophobic barnacle and foulingrelease performance.

\section{Acknowledgements}

This work was supported by National nature science foundation of China (51179018), defense fund and the application basic research fund of the Ministry of Transport (2013329225330). The authors gratefully acknowledge for financial support.

\section{References}

1. Elisabete A., Teresa C. D., Orlando de S, Prog. Org. Coat., 59, 2-20(2007)

2. D. M. Yebra, S. Kiil, K. Dam-Johansen. Prog. Org. Coat., 50, 75 (2004)

3. T. Vladkova, Journal of the University of Chemical Technology and Metallurgy, 42,239(2007)

4. Chambers L.D., Stokes K.R., Walsh F.C., et al. Surface \& Coatings Technology, 201, 36423652(2006)

5. Yu-Hong Qi, Zhan-Ping Zhang, Hui Gao, Proc. of SPIE, 7493,74934M(2009)

6. Shan Cao, Jiadao Wang, Darong Chen, Microbial Ecology, 62(4): 931-940(2011)

7. R.E. Baier, E.G. Shafrin, W.A. Zisman, Science, 162, 1360-1368 (1968)

8. R.F. Brady, Prog. Org. Coat. 43,188-192 (2001)

9. T.J. Gui, Ocean University of China, Tsingtao, (2008)

10. Zhanping ZHANG, Yuhong QI, Miao BA, et al. Advanced Materials Research, 842, 737-741(2014) 